\title{
Paper A Dual-band Circularly-polarized Printed Monopole Antenna for Wi-Fi and WiMAX Applications
}

\author{
Zaw Myo Lwin ${ }^{1}$ and Thae Su Aye ${ }^{2}$ \\ ${ }^{1}$ Department of Electronic Engineering, Technological University (Kyaukse), Myanmar \\ ${ }^{2}$ Faculty of Electronic Engineering, University of Technology (Yatanarpon Cyber City), Myanmar
}

https://doi.org/10.26636/jtit.2019.136719

\begin{abstract}
This paper presents a rectangular-shaped printed monopole antenna with circular polarization for $\mathrm{Wi}-\mathrm{Fi}$ (2.4-2.484 GHz) and WiMAX (3.3-3.7 GHz) bands. The antenna relies on asymmetric arrangement of the patch with respect to the microstrip feed, in order to generate circular polarization. Dual-band (Wi-Fi and WiMAX) operation is enabled by inserting a slit in the corner of the ground plane. Simulation results show a bandwidth increase of $15.9 \%$ (2.2-2.58 GHz) for Wi-Fi, and of $24.16 \%(3.13-3.99 \mathrm{GHz})$ for WiMAX applications. Furthermore, beamwidths at the axial ratio of $3 \mathrm{~dB}$ equal $48^{\circ}$ and $51^{\circ}$ for the $x-z$ plane and $y-z$ planes, respectively.
\end{abstract}

Keywords-circular polarization, dual-band antenna, printed monopole antenna, rectangular slit.

\section{Introduction}

Circularly-polarized (CP) antennas offer a number of advantages compared to their linearly-polarized counterparts, because they are able to overcome fading, mitigate the Faraday rotation effect and provide a nearly-fixed received signal level independent of the aerial position [1]. Therefore, they are gaining popularity in wireless communication systems. With multiband CP operations, instead of a number of single-band $\mathrm{CP}$ antennas, deployment of one multiband $\mathrm{CP}$ antenna is a much better choice [1].

Many designs have been proposed for dual-band and multiband printed monopole antennas with circular polarization - e.g. [2]-[6]. In [7], a wideband printed rectangular monopole antenna for circular polarization has been proposed. However, as compared to [7], this paper sets out to reduce the size and widen the beamwidth by proposing a dual-band CP antenna with a rectangular slit in the ground plane. The factor that differentiates the solution from that presented in [7] is related to the geometry of the antenna and consists in the fact that its dimensions are optimized for the desired frequency bands. In addition, a slit is inserted at the corner of the ground plane, enabling a dual-band operation. The antenna dimensions are $51 \times 51 \times 3.2 \mathrm{~mm}$ and the inserted rectangular slit has the length of $16.5 \mathrm{~mm}$ and the width of $1 \mathrm{~mm}$.
The results show an enhancement in the beamwidth at the axial ratio of $3 \mathrm{~dB}$, compared with the design presented in [7].

The following sections of the paper present the antenna structure, the principle of circular polarization and the simulated results.

\section{The Antenna Design}

The proposed antenna design is illustrated in Fig. 1. The dielectric has a span of $D^{\prime}=D^{\prime \prime}=51 \mathrm{~mm}$. The rectangular patch, which is tilted at 45 -degrees with respect to the antenna's y axis, has the width of $W=20.56 \mathrm{~mm}$ and the length of $L=36 \mathrm{~mm}$. The ground plane, which is positioned on the other side of the dielectric, has a the dimensions of $B_{1}=22 \mathrm{~mm}, B_{2}=32.8 \mathrm{~mm}$ and $B_{3}=40 \mathrm{~mm}$. Although positioned at different layers, just for the scaling, the left- and right-hand side spacing between the patch and the ground plane measure $3.1 \mathrm{~mm}$ and $2 \mathrm{~mm}$, respectively. The microstrip feed line, with the width of $w=6 \mathrm{~mm}$ width, meets the patch at a distance of $V=5.4 \mathrm{~mm}$ away from the bottom corner of the patch. The feed point and the patch's bottom corner are positioned at $U=3 \mathrm{~mm}$ and $X=7.4 \mathrm{~mm}$, respectively, from the lower edge of the dielectric, which has the thickness of $3.2 \mathrm{~mm}$, the relative permittivity $\varepsilon_{r}$ of 4.7 and the loss tangent of 0.02. Similar to [7], the rectangular patch is kept asymmetric to the microstrip feed line, so that circular polarization can be produced. The antenna's designed dimensions are listed in Table 1.

In order to enable dual-band operation, the slit is inserted from the left corner of the ground plane and is directed towards the feed point. Length S1 and width S2 of the slit are determined by parametric studies. However, the length of the slit can be approximated by:

$$
S_{1} \approx \frac{\lambda_{g}}{4}=\frac{c}{4 f \sqrt{\frac{\varepsilon_{r}+1}{2}}},
$$

where $\lambda_{g}$ is the guided wavelength and $f$ is the operating frequency. 
(a)

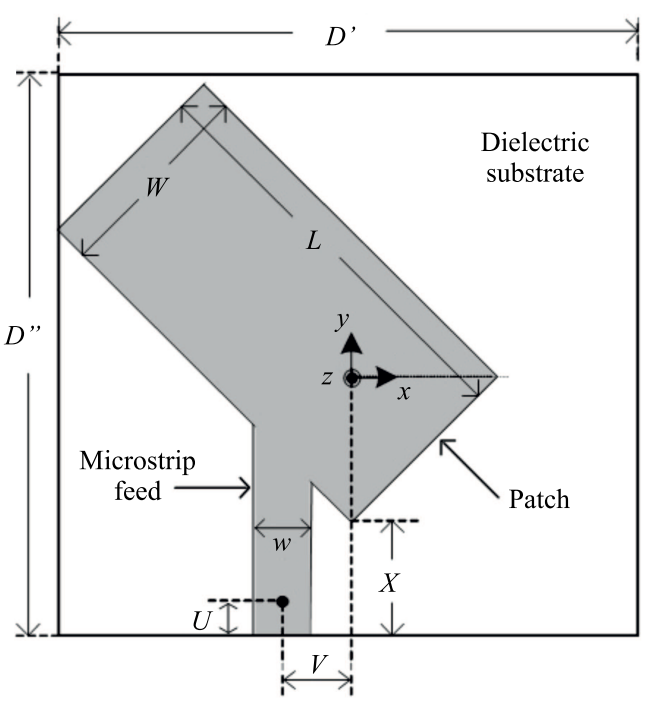

(b)

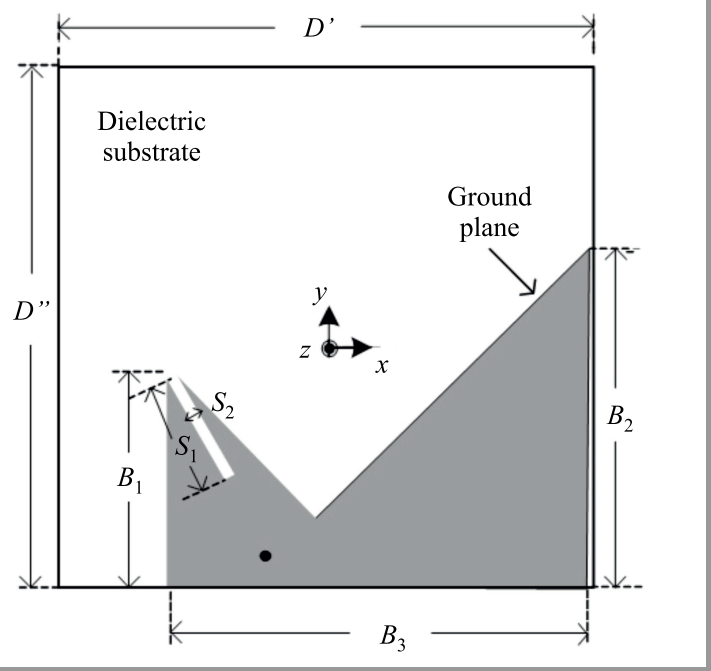

Fig. 1. The proposed antenna structure: (a) top view, (b) bottom view.

Table 1

Designed parameters of the antenna

\begin{tabular}{|c|c|c|c|}
\hline Parameter & Value $[\mathrm{mm}]$ & Parameter & Value $[\mathrm{mm}]$ \\
\hline \hline$D^{\prime}, D^{\prime \prime}$ & 51 & $X$ & 7.4 \\
\hline$L$ & 36 & $B_{1}$ & 22 \\
\hline$W$ & 20.56 & $B_{2}$ & 32.8 \\
\hline$w$ & 6 & $B_{3}$ & 40 \\
\hline$U$ & 3 & $S_{1}$ & 16.5 \\
\hline$V$ & 5.4 & $S_{2}$ & 1 \\
\hline
\end{tabular}

\section{Proof of Circular Polarization}

The current distribution at the frequency of $2.43 \mathrm{GHz}$ (Wi-Fi) is shown in Fig. 2. At $\omega t=10^{\circ}$, the electric currents on both the patch and the ground plane flow from the bottom right to the top left. At this instant, since the current on the left-hand side of the ground plane is stronger, it is the source of the electric field radiation. Mea-

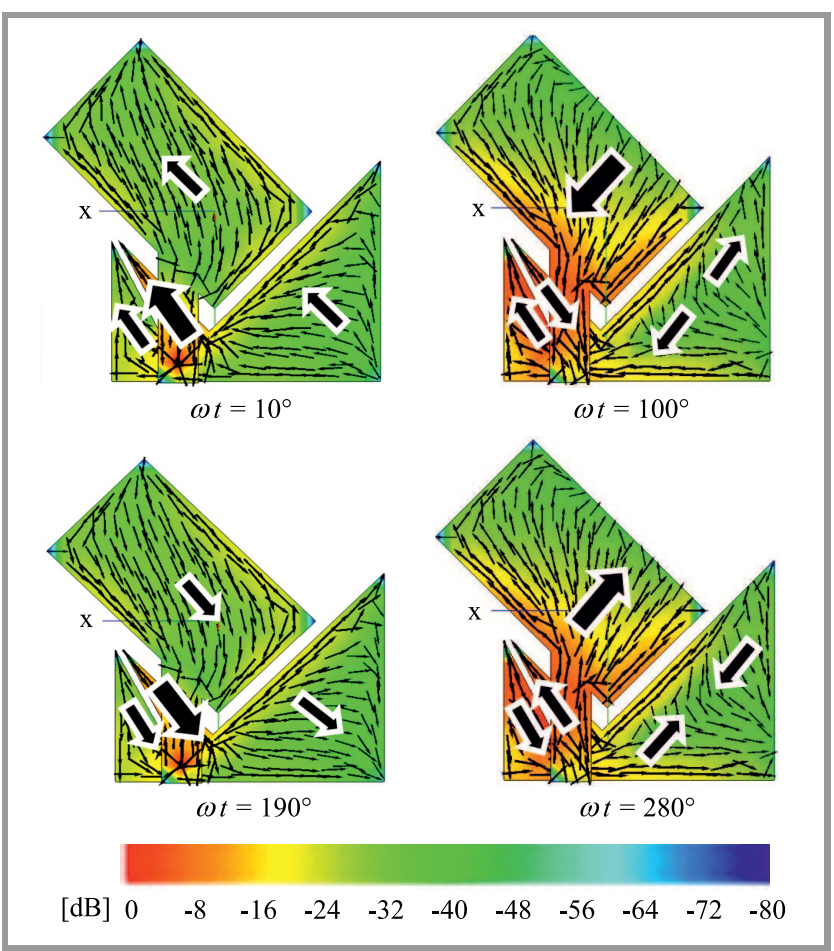

Fig. 2. Electric current distributions at $2.43 \mathrm{GHz}$. (For color pictures, visit www.nit.eu/publications/journal-jtit)

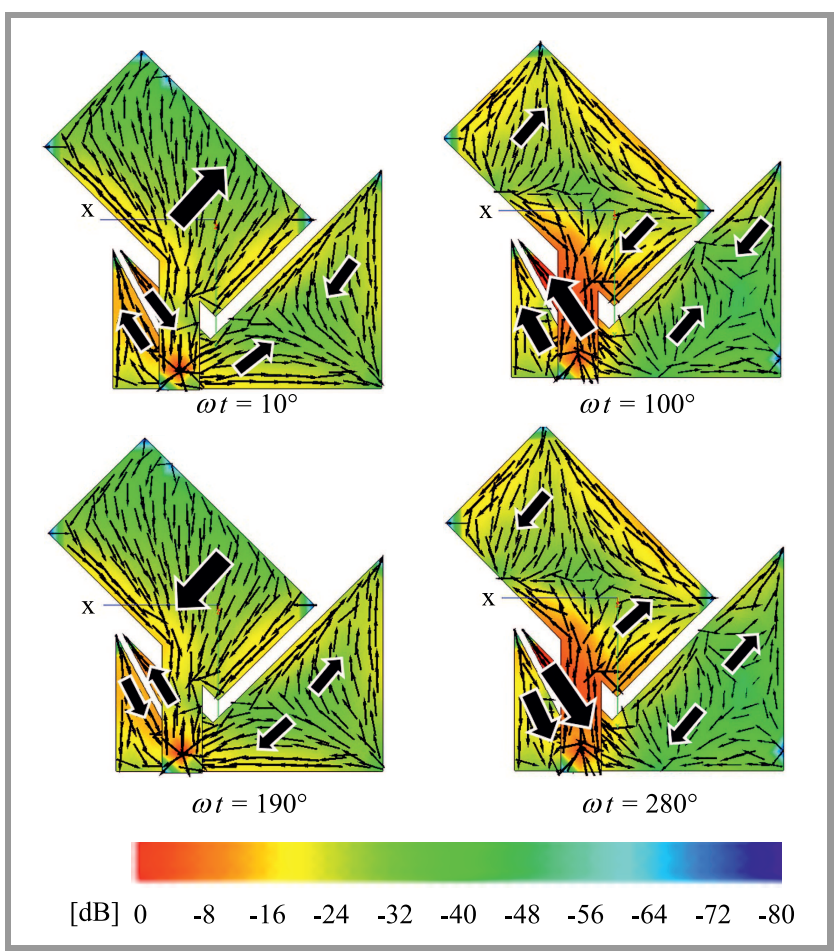

Fig. 3. Electric current distributions at $3.57 \mathrm{GHz}$.

sured from the positive $\mathrm{x}$ axis, the electric field direction is about $135^{\circ}$. At $\omega t=100^{\circ}$, the electric currents on the left-hand side of the ground plane flow in the other direction, similarly to the currents on the right-hand side of the ground plane. As a result, there is no radiation from the ground plane. Only the current on the patch, which 
flows from the top right to the bottom left, plays a major role for electric field radiation, with its direction approximated at $-135^{\circ}$. The electric current distribution at $\omega t=$ $190^{\circ}$ and $\omega t=280^{\circ}$ is similar, as $\omega t=10^{\circ}$ and $\omega t=100^{\circ}$, respectively, in spite of the currents flowing in different directions. The associated electric field directions are $-45^{\circ}$ and $45^{\circ}$ for $\omega t=190^{\circ}$ and $\omega t=280^{\circ}$, respectively.

Figure 3 depicts electric current distribution at $3.57 \mathrm{GHz}$ (WiMAX). At $\omega t=10^{\circ}$, both currents on the left-hand side and on the right-hand side of the ground plane flow in opposing directions, rendering their effects negligible. Therefore, the current on the patch flowing from the bottom left to the top right creates an electric field radiation directed at about $45^{\circ}$. Again, at $\omega t=100^{\circ}$, the current on the patch and on the right-hand side of the ground plane flow in opposite directions, so they become insignificant for aerial radiation. Only the current on the left-hand side of the ground plane, flowing towards the top left, is the source generating electric field radiation of approximately $135^{\circ}$. In spite of flowing in opposite directions, the electric currents at $\omega t=190^{\circ}$ and $\omega t=280^{\circ}$ have the same distribution as those at $\omega t=10^{\circ}$ and $\omega t=100^{\circ}$, respectively. The corresponding electric field directions are $-135^{\circ}$ for $\omega t=190^{\circ}$ and $-45^{\circ}$ for $\omega t=280^{\circ}$. As seen in Figs. 2 and 3 , the electric field rotation with time is quite obvious and, hence, a circularly-polarized wave is produced at both frequency bands.

\section{Results and Discussion}

Further research has been performed to identify the effect of the inserted slit on the antenna's performance, by varying length $S_{1}$ and the width $S_{2}$. Figure 4 shows the simulated return loss and axial ratio of the designed antenna as a function of the slit length. For $16 \mathrm{~mm}$, both return loss and axial ratio at the lower band are better than for two other lengths, but performance is poor at the higher band. With a $17 \mathrm{~mm}$ slit, the antenna exhibits a better return loss and improved axial ratio performance at the higher band, but offers poor characteristics in the lower band. Unlike the two length values mentioned above, slit length of $16.5 \mathrm{~mm}$ performs satisfactorily in both frequency bands. Hence, $S_{1}=16.5 \mathrm{~mm}$ is chosen.

Figure 5 shows the simulated return loss and axial ratio for different slit widths. Slit widths of $0.5 \mathrm{~mm}$ and $1.5 \mathrm{~mm}$ provide acceptable return loss characteristics in both bands, but these values cannot provide good axial ratio performance for both bands. Only the slit width of $1 \mathrm{~mm}$ outperforms the other two in both bands, both in with respect to return loss and axial ratio characteristics. Therefore, the slit width of $1 \mathrm{~mm}$ is the optimized width for the proposed antenna. One may notice from Figs. 4 and 5 that the frequency bandwidths (to satisfy a $10 \mathrm{~dB}$ return loss and a $3 \mathrm{~dB}$ axial ratio) are $15.9 \%(2.2-2.58 \mathrm{GHz})$ for $\mathrm{Wi}-\mathrm{Fi}$ band and $24.16 \%(3.13-3.99 \mathrm{GHz})$ for WiMAX frequency bands, respectively.
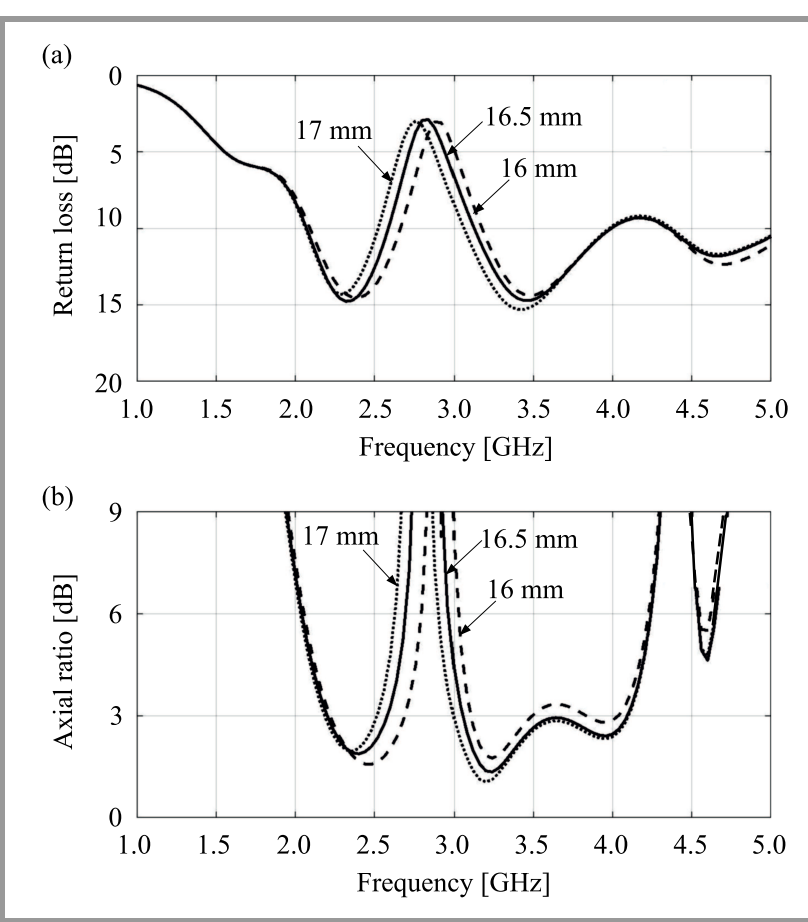

Fig. 4. Simulation of: (a) return loss and (b) axial ratio as a function of the slit length $S_{1}$.

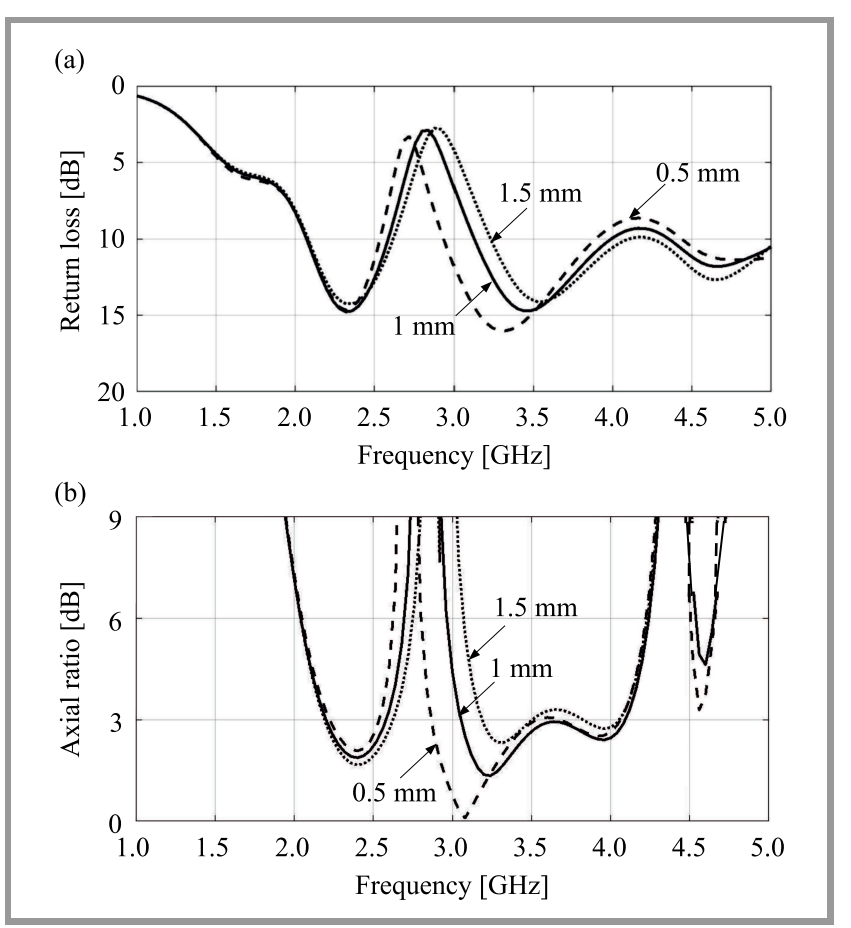

Fig. 5. Simulation of: (a) return loss and (b) axial ratio for three slit width $S_{2}$ values.

The simulated total antenna gain is depicted in Fig. 6. It equals approximately $1 \mathrm{dBi}$ in the $\mathrm{Wi}-\mathrm{Fi}$ band and above $-2.8 \mathrm{dBi}$ in the WiMAX band. Although the gain is nearly flat in the Wi-Fi band, there is a gain slope in the WiMAX band, which should be improved in the future. One of the main possible causes of the low gain values is the use of FR4 laminate with a relatively high loss tangent (0.02) 


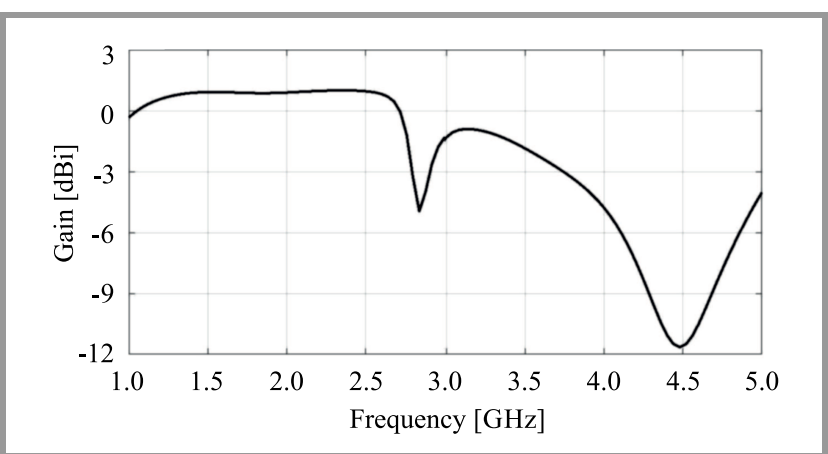

Fig. 6. Total antenna gain.

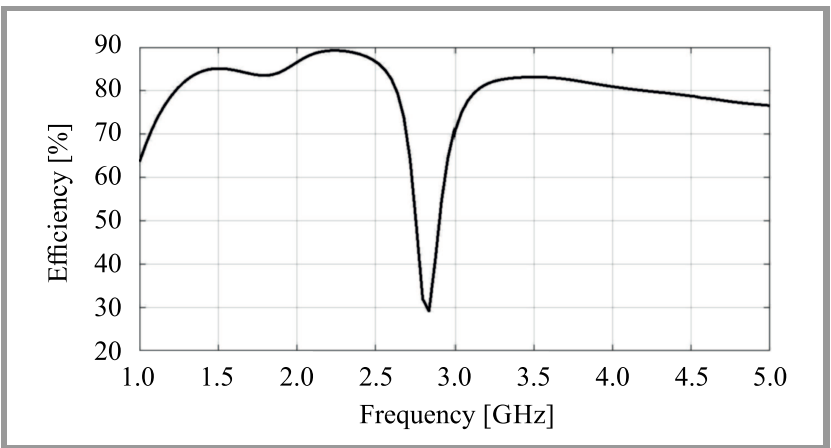

Fig. 7. Efficiency of the proposed antenna.

which translates to poor performance at high frequencies. Although the gain is low in the higher WiMAX band, the simulated efficiency of the proposed antenna is well above $80 \%$ in both bands. As shown in Fig. 7, the efficiency is approximately $87 \%$ in the lower band and approximately $83 \%$ in the higher band.

The normalized radiation patterns for $2.43 \mathrm{GHz}$ and 3.57 $\mathrm{GHz}$ are shown in Fig. 8. Good radiation characteristics for circular polarization can be observed in both Wi-Fi and WiMAX bands, although radiations pattern for the lower band are relatively better than those for the higher band. According to the results, right-hand circular polarization (RHCP) radiation is generated along the direction of the positive $\mathrm{z}$ axis, while left-hand circular polarization (LHCP) radiation is generated along the negative $\mathrm{z}$ axis.

The beamwidth measured at the axial ratio of $3 \mathrm{~dB}$ for the proposed antenna and the $y-z$ design from [7] are depicted in Fig. 9. At $2.43 \mathrm{GHz}$, the antenna [7] has a beamwidth of $28^{\circ}$ and $24^{\circ}$ in the $x-z$ plane and $y-z$ plane, respectively, with the beamwidth of $48^{\circ}$ achieved in the $x-z$ plane and of $51^{\circ}$ in the $y-z$ plane. Therefore, it is observed that the proposed antenna provides a wider beamwidth than [7]. At $3.57 \mathrm{GHz}$, the proposed antenna has the beamwidth, at the axial ratio of $3 \mathrm{~dB}$, of $10^{\circ}$ for the $\mathrm{x}-\mathrm{z}$ plane and $8^{\circ}$ for the $y-z$ plane. This result is not compared with [7], because $3.57 \mathrm{GHz}$ (WiMAX) band is beyond the bandwidth of the design from [7].

The overall dimension of [7] is $0.57 \lambda \times 0.57 \lambda$ at $2.43 \mathrm{GHz}$ - a little bit more than the size of the proposed antenna equaling $0.41 \lambda$. Therefore, the proposed antenna is fairly smaller than the one in [7]. (a)
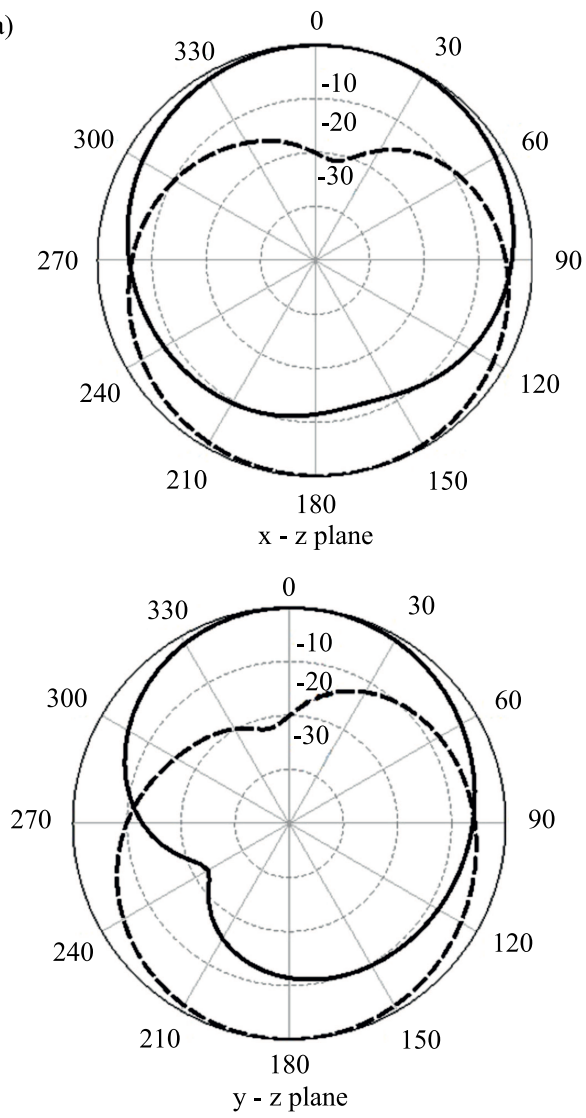

(b)
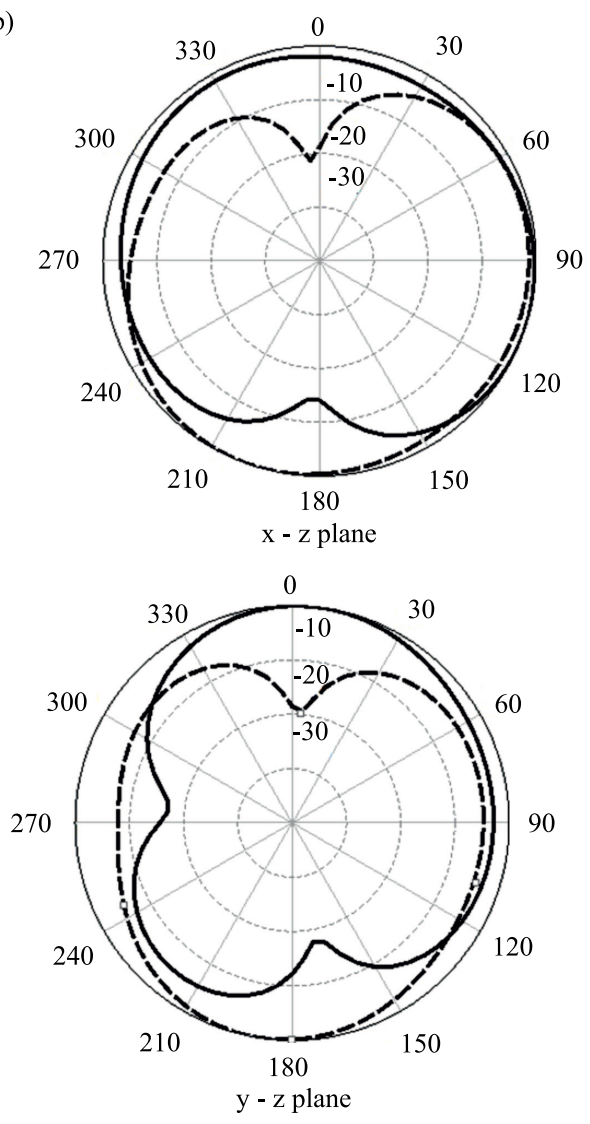

Fig. 8. Normalized RHCP (solid) and LHCP (dashed) radiation patterns (in $\mathrm{dB}$ ): (a) $2.43 \mathrm{GHz}$ and (b) $3.576 \mathrm{GHz}$. 


\section{Conclusion}

This paper presents a circularly-polarized rectangular printed monopole antenna for Wi-Fi and WiMAX wireless communication systems.

The simulated results shown in this paper prove that the insertion of a slit in the ground plane enables the dualband operation and that circular polarization was achieved as well. Smaller size and wider beamwidth were obtained thanks to the work described in this paper, compared to the antenna presented in [7]. It may be concluded that the achieved bandwidth (required to satisfy a $10 \mathrm{~dB}$ return loss and a $3 \mathrm{~dB}$ axial ratio) of $15.9 \%(2.2-2.58 \mathrm{GHz})$ and $24.16 \%(3.13-3.99 \mathrm{GHz})$ is suitable for circularly-polarized Wi-Fi and WiMAX applications.

\section{References}

[1] S. Gao, Q. Luo, and F. Zhu, Circularly Polarized Antennas. WileyIEEE Press, 2013 (ISBN: 978-1118374412).

[2] Z. Liang, Y. Li, and Y. Long, "Multiband monopole mobile phone antenna with circular polarization for GNSS application", IEEE Trans. on Antenn. and Propag., vol. 62, no. 4, pp. 1910-1917, 2014 (doi: 10.1109/TAP.2014.2299821).

[3] H. Zhang, Y. C. Jiao, W. L. Liang, and L. Lu, "Dual-band circularly polarized deformed monopole antenna", Progress in Electromag. Res. $C$, vol. 54, pp. 117-124, 2014 (doi: 10.2528/PIERC14100603).

[4] H. C. Park, "Very simple 2.45/3.5/5.8 GHz triple-band circularly polarised printed monopole antenna with bandwidth enhancement", Electron. Lett., vol. 50, no. 24, pp. 1792-1793, 2014 (doi: 10.1049/el.2014.2935).

[5] J. H. Lu and C. W. Liou, "Planar dual-band circular polarization monopole antenna for wireless local area networks", IEEE Antenn. and Wirel. Propag. Lett., vol. 14, pp. 478-481, 2015 (doi: 10.1109/LAWP.2014.2368137).

[6] M. T. Tan and B. Z. Wang, "A dual-band circularly polarized planar monopole antenna for WLAN/Wi-Fi applications", IEEE Antenn. and Wirel. Propag. Lett., vol. 15, pp. 670-673, 2016 (doi: 10.1109/LAWP.2015.2466596).

[7] T. Fujimoto and K. Jono, "Wideband rectangular printed monopole antenna for circular polarisation", IET Microw., Antenn. \& Propag., vol. 8, no. 9, pp. 649-656, 2014 (doi: 10.1049/iet-map.2013.0460).

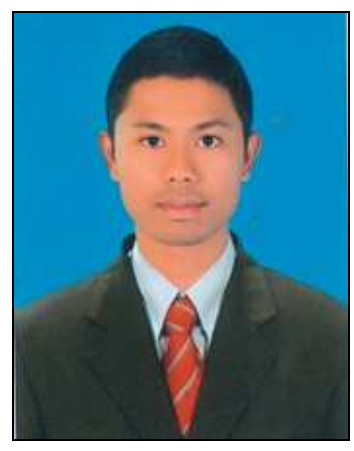

Zaw Myo Lwin received his Bachelor of Engineering (B.E.) degree in Electronics from Mandalay Technological University, Myanmar in 2004. Then, he received the Master of Engineering (M.E.) degree in Electronics from Yangon Technological University, Myanmar in 2006. In 2011, he received his $\mathrm{Ph} . \mathrm{D}$. degree in Electronic Engineering from Mandalay Technological University, Myanmar. From 2008 to 2010, he was building his research experience in optical fiber communications at University of ElectroCommunications, Tokyo, Japan. Currently, his main interests are the design of wideband and multiband CP printed antennas.

Email: zawmyolwinn@gmail.com

Department of Electronic Engineering

Technological University (Kyaukse)

Kyaukse, Mandalay Division, Myanmar

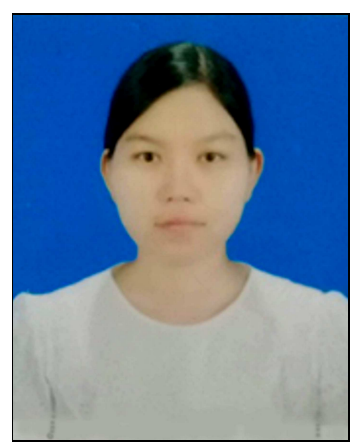

Thae Su Aye received the Bachelor of Engineering (B.E.) degree in Electronics from Mandalay Technological University, Myanmar in 2004. Then, she received the Master of Engineering (M.E.) degree in Electronics from Yangon Technological University, Myanmar in 2006. In 2011, she received her Ph.D. degree in Electronic Engineering from Mandalay Technological University, Myanmar. Her research interests are in microstrip filters and high frequency circuit designs.

Email: thaesuaye1483@gmail.com

Faculty of Electronic Engineering

University of Technology (Yatanarpon Cyber City)

Mandalay Division, Myanmar 